Inhibition in motor memory

\title{
EEG beta power increase indicates inhibition in motor memory
}

\author{
Running head: Inhibition in motor memory \\ Tobias Tempel ${ }^{1}$, Christian Frings ${ }^{2}$, and Bernhard Pastötter ${ }^{2}$ \\ ${ }^{1}$ Ludwigsburg University of Education \\ ${ }^{2}$ University of Trier
}

Preprint.

Accepted in the International Journal of Psychophysiology:

Tempel, T., Frings, C., \& Pastötter, B. (2020). EEG beta power increase indicates inhibition in motor memory. International Journal of Psychophysiology, 150, 92-99.

DOI: https://doi.org/10.1016/j.ijpsycho.2020.02.008 
Inhibition in motor memory

\section{Abstract}

Beta power increase has been suggested to be an electrophysiological marker of response inhibition during voluntary action stopping. We examined whether beta power increase accompanies inhibition in human motor memory, focusing the phenomenon of retrieval-induced forgetting that has been assumed to be the consequence of inhibition in memory. Whereas most studies on this effect comprise word materials, a variant of the retrieval-induced-forgetting paradigm exists for examining motor memory. In the present study, we recorded scalp EEG during this motor-specific variant and examined EEG oscillatory correlates of retrieval-induced slowing of motor actions. Here, forgetting occurred in the form of significant slowing when executing motor sequences in a final recall test. Participants first learned to associate memory cues to sequential finger movements, half of which were performed with the left hand and half with the right hand. They then selectively retrieval-practiced half of the items of one hand before finally memory for all items was tested. On a behavioral level, selective retrieval practice induced slower execution of the nonretrieved items of the retrieval-practiced hand in a final memory test. On a physiological level, this retrieval-induced-slowing effect was predicted by an increase of EEG beta power during retrieval practice. The results suggest that motor retrieval-induced forgetting is a consequence of inhibition in human memory.

Keywords: memory retrieval; body movement; retrieval-induced forgetting; EEG 
Inhibition in motor memory

\section{Highlights}

- Inhibition is a heavily debated concept in cognitive research.

- The existence of inhibitory mechanisms in motor control is mostly accepted.

- Evidence on inhibition in memory is more controversial.

- The current experiment combined the motor domain and the memory domain.

- The results suggest an inhibitory mechanism in motor memory. 
Inhibition in motor memory

Motor memory bridges two domains that are typically researched in separation from each other: memory and motor action. A focus on motor memory permits examining commonalities in both domains, such as, the operation of inhibitory mechanisms and their physiological correlates. In the present investigation, we investigated a memory phenomenon (retrieval-induced forgetting) that has been linked to the concept of inhibition on the one hand and was shown to affect motor sequences stored in memory on the other hand.

Interference between body movements arises on different levels. For example, when an ongoing action impairs the response to a sudden demand or when habitual behavior disrupts the acquisition of novel motor sequences. The human mind possesses executive control mechanisms to handle such interference. In particular, voluntary suppression of motor action has received much attention (in the go/no-go and stop-signal paradigm; for reviews see Verbruggen \& Logan, 2008; Wessel, 2018) and inspired research also beyond the motor domain (e.g. Anderson \& Green, 2001). Mostly, an inhibitory mechanism is considered to underlie the ability to stop the execution of a motor response after its initiation (response inhibition; e.g. Friehs \& Frings, 2018). Thus, motor action typically is considered a domain that involves executive control through inhibition, whereas in other (cognitive) domains, such as, attention and memory, the existence of inhibitory mechanisms is strongly debated (e.g., Wühr \& Frings, 2008).

Research on retrieval-induced forgetting in large part has centered on the question whether this phenomenon reflects inhibition in human memory (see Bäuml, Pastötter, \& Hanslmayr, 2010). Retrieval-induced forgetting occurs when the selective retrieval of a subset of information reduces accessibility of the non-retrieved rest of information from that set. It is typically analyzed in a paradigm that consists of three main phases (Anderson, Bjork, \& Bjork, 1994). In the study phase, participants encode several sets of items in combination with a shared (category-)cue that defines the specific set of items. In the 
Inhibition in motor memory

retrieval-practice phase, participants then are cued to recall half of the studied items from half of the sets. In the test phase, recall performance for all items is tested. Recall of practiced items ( $\mathrm{Rp}+)$, non-practiced items from practiced sets ( $\mathrm{Rp}-)$, and non-practiced items from non-practiced sets $(\mathrm{Nrp})$ is compared. Whereas $\mathrm{Rp}+$ items normally profit from retrieval practice and are better recalled than Nrp items, retrieval-induced forgetting manifests itself in lower recall of Rp- as compared to Nrp items.

Tempel and Frings (2013) adapted this paradigm for the use of motor sequences as item material. Participants study a certain number of sequential finger movements that consist of two or more key presses each. These motor sequences are learned as responses to individual stimuli (e.g., letters or numbers). Half of the sequences are performed with the left hand, half with the right hand. In the retrieval-practice phase, participants are cued to recall half of the motor sequences learned with one hand $(\mathrm{Rp}+)$. In a final test, this retrieval practice results in worse recall performance, i.e., reduced accuracy and/or increased response time, for the remaining items of the practiced hand ( $\mathrm{Rp}-)$ compared to items of the other, not practiced hand (Nrp).

Subsequent studies documented several properties of motor retrieval-induced forgetting that matched key predictions of the inhibition theory. Selective restudy in lieu of retrieval practice did not impair memory for the non-practiced rest of items from that set (retrieval specificity; Tempel \& Frings, 2016; Tempel, Loran, \& Frings 2015) and the occurrence of retrieval-induced forgetting depended on interference between $\mathrm{Rp}+$ and $\mathrm{Rp}$ items (competition dependence; Tempel, Aslan, \& Frings, 2016), whereas it was independent from the amount of strengthening of Rp+ items (strength independence; Tempel \& Frings, 2017). In addition, we identified motion-specific features. Motor retrieval-induced forgetting affects motor programs (Tempel \& Frings, 2014a) and can arise based on an organization of motor sequences by body parts (Tempel \& Frings, 2014b), by movement direction (Tempel 
Inhibition in motor memory

\& Frings, 2015), or by location (Reppa, Worth, Greville, \& Saunders, 2013; Tempel \& Frings, 2017).

One peculiarity of retrieval-induced forgetting in the motor domain is that it can appear in the form of retrieval-induced slowing. Our first study, for example, found only in two out of three experiments that selective retrieval practice reduced the number of correctly recalled motor sequences but in all three experiments the execution speed was significantly reduced (Tempel \& Frings, 2013). Moreover, retrieval-induced slowing of Rp- motor sequences has been shown to occur in indirect memory tests as well that did not rely on the recall of previously encoded sequences but an enactment of corresponding movements in response to novel cues, thereby tapping the same motor program (Tempel \& Frings, 2014a, 2015). We observed that movements were executed significantly more slowly when they matched Rp- items as compared to Nrp items. Such slowing may suggest that procedural memory representations of the motor sequences had been affected by retrieval practice. The storage of motor programs is a specific property of using motor sequences as item material. In fact, tasks that serve the investigation of motor learning (e.g. the Serial Reaction Time Task; Willingham, Nissen, \& Bulemer, 1989) typically focus on accelerated execution as an index of learning. Correspondingly, slowing can be regarded as an index of forgetting (see also Reppa et al., 2013; Wohldmann, Healy, \& Bourne, 2008).

In the present study, we tested the inhibition theory by investigating motor-specific EEG signals in the motor retrieval-induced forgetting paradigm. Although several results of the previously reported behavioral work on motor retrieval-induced forgetting were consistent with postulates of the inhibition theory, those findings can be understood as conceptual replications of corresponding results from studies that examined retrieval-induced forgetting with the more common material of word pairs. However, further ways of testing predictions of the inhibition theory of retrieval-induced forgetting are necessary because it 
Inhibition in motor memory

has been argued repeatedly that behavioral predictions of the inhibition theory can be conciliated with non-inhibitory theories in many cases (Jonker, Seli, \& MacLeod, 2013; Raaijmakers \& Jakab, 2013; Raaijmakers, 2018), either by supplementary assumptions or methodological criticism. EEG oscillatory brain signals can be used to test the inhibition theory of retrieval-induced forgetting on physiological grounds by examining in which way oscillatory patterns during retrieval practice predict retrieval-induced forgetting.

According to the inhibition theory of retrieval-induced forgetting (Anderson, 2003), the purpose of inhibition is facilitating retrieval by reducing interference. In correspondence with this assumption, Staudigl, Hanslmayr, and Bäuml (2010) observed that midfrontal EEG theta power, which is a prominent marker of cognitive interference (see Nigbur et al., 2011), decreased when selective retrieval of word material was repeated over two retrieval-practice cycles in the retrieval-induced forgetting paradigm. In addition, Staudigl et al. (2010) demonstrated that the increase of theta power $(5-9 \mathrm{~Hz})$ over retrieval-practice cycles was source localized to the anterior cingulate cortex, a brain region that has also been linked to interference and retrieval-induced forgetting in previous imaging work (Kuhl, Dudukovic, Kahn, \& Wagner, 2007; Wimber, Rutschmann, Greenlee, \& Bäuml, 2008). In support of the inhibition theory of retrieval-induced forgetting, Waldhauser, Johansson, and Hanslmayr (2012) examined lateralized EEG power effects during selective retrieval of nonverbal, visual target information, which was studied together with irrelevant distractor information. All information was presented lateral to fixation during original study. During selective retrieval, reliable EEG alpha/beta power increase was observed contralateral to distractor presentation side, which was suggested to reflect retrieval-induced inhibition of visual memories. In the study by Waldhauser et al. (2012), no significant correlation between midfrontal theta power and lateralized alpha/beta power was observed. 
Inhibition in motor memory

The present study of motor retrieval-induced forgetting followed this perspective on the role of inhibition in retrieval-induced forgetting by making use of both EEG theta oscillations, a prominent interference marker in both motor and non-motor domains, and motor-related beta oscillations, a prominent motor-specific inhibition marker. Indeed, beta power increase has been shown to be a reliable marker of response inhibition in various motor tasks (e.g. Alegre et al., 2004; Pastötter, Hanslmayr, \& Bäuml, 2008; Swann et al., 2009; Wheaton et al., 2009). Moreover, an increase of beta power has also been considered as reflecting reduced information processing, which could be a consequence of inhibition (Hanslmayr et al., 2012; Waldhauser et al., 2012). Assuming that EEG beta power increase relates to both response inhibition and inhibition in motor memory, in the present study, we expected to find a beta power increase during repeated retrieval practice of motor sequences when comparing the first half with the second half of practice cycles. Moreover, we expected that beta power increase predicts behavioral motor retrieval-induced forgetting. In addition, we expected to replicate the finding by Staudigl et al. (2010) of a reduction of theta power over retrieval-practice cycles, as well.

\section{Method}

Participants. Fifty-nine undergraduate students at the University of Trier participated in the experiment. All participants were right-handed, reported normal or corrected-to-normal vision, gave written informed consent before examination, and received course credit for participation or were paid 20 Euro. Eleven participants did not enter data analysis because of neurological abnormalities (three), abortion of the experiment (four), or not following task instructions (four). Thus, 48 participants ( 38 females, 10 males; mean age $=22.9$ years, standard deviation $=3.2$ years) were included in data analysis. The study was conducted in accordance with the Declaration of Helsinki and approved by the local ethical review committee at the University of Trier (reference number: 50/2017). 
Inhibition in motor memory

Design. Item type (Rp+, Rp-, Nrp-, Nrp+) was manipulated within participants. Rpitems were tested before $\mathrm{Rp}+$ items to preclude any output interference by $\mathrm{Rp}+$ items (cf. Anderson et al., 1994; Roediger \& Schmidt, 1980; Smith, 1971). Rp- items accordingly were compared to the first three Nrp items tested (Nrp-), whereas Rp+ items were compared to the last three Nrp items tested $(\mathrm{Nrp}+)$.

Material. The experiment was conducted in a soundproof test cabin. The software EPrime 2.0 (SP 2) served for running the experiment.

The items consisted of 24 sequential finger movements to be performed either with the left index finger or with the right index finger (each comprising three key presses; see Appendix). The right-hand movements were performed by pressing three marked keys on the right side of the keyboard (1,2, and 3 within the numeric keypad), the left-hand movement by pressing three marked keys on the left side of the keyboard $(Y, X$, and $C)$.

Procedure. The experiment consisted of two main blocks that each comprised four phases (study, retrieval practice, distractor task, test). The two blocks were identical with each other except for the items and response hand in the retrieval-practice phase of the experiment.

Instructions were given on the screen and summarized by an experimenter. In study trials, first the category was indicated by the word rechts or links (English: right or left) that was displayed in the center of the screen. Participants pressed the middle key to start a study trial. After showing a fixation cross for $2,500 \mathrm{~ms}$, a letter (or, respectively, a number in block 2) appeared in the center of the screen together with three squares representing the three response keys (cf. Figure 1, upper section). After 1,500 ms, an animation of consecutively red illuminating squares started demonstrating which keys should be pressed (200 ms per illumination, separated by displays of the uncolored squares for $200 \mathrm{~ms})$. Subsequently, the three squares disappeared and participants could perform the movement. If the performed 
Inhibition in motor memory

sequence was incorrect, feedback appeared, displaying: Fehler! (English: Error!). Study trials were separated by intervals of 1,000 ms. First, all 12 items appeared once in random order, whereupon an instruction screen informed the participant that the items just presented would appear again several times in the following. Ten cycles were presented each containing the twelve items in a random order, under the constraint that the same hand was not repeated more than three times in a row.

The retrieval-practice phase cued participants to recall half (i.e. three) of the motor sequences of one hand by presenting the corresponding stimuli. Participants had to enter the motor sequence via the same response keys as in the study phase. At the beginning of each trial, the word weiter (English: continue) was displayed in the center of the screen. Upon pressing the middle button, a fixation cross appeared for 2,500 ms. Subsequently, the stimulus of a sought item appeared (cf. Figure 1, lower section). No feedback about the accuracy of the performed movement was given. We counterbalanced between participants which three items received retrieval practice. Items were practiced in ten cycles. In analyses of behavioral data as well as EEG signals the first half (i.e. the first five cycles) were compared with the second half (i.e. the last five cycles). 


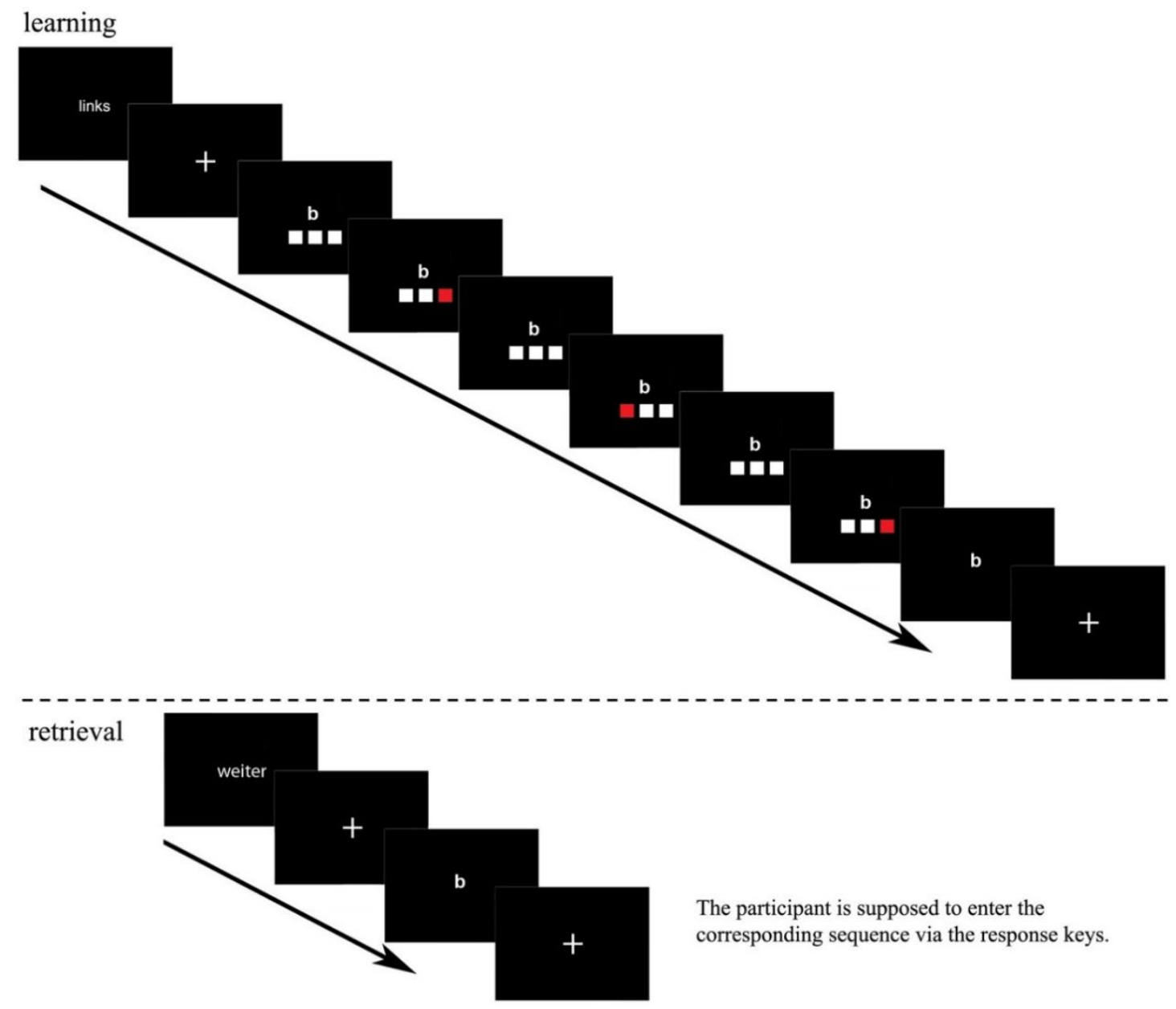

Figure 1. The upper section depicts a study trial. First, the hand is indicated (English: left). After pressing the middle of the three left response keys with the left index finger, a fixation cross is shown. After 2,500 ms, a letter stimulus appears together with three squares representing the three response keys. A first square illuminates after $1,500 \mathrm{~ms}$ in red color for $200 \mathrm{~ms}$, then a second square illuminates for $200 \mathrm{~ms}$, and then a third square illuminates for $200 \mathrm{~ms}$. The illuminations are separated by intervals of $200 \mathrm{~ms}$ showing the uncolored squares. Subsequently, the participant can enter the sequential finger movement just illustrated. The lower section depicts a trial in the retrieval-practice and the test phase. First, the word weiter (English: continue) is displayed. After pressing the middle of the three left response keys with the left index finger, a fixation cross is shown. After 2,500 ms, a stimulus is given and the participant is supposed to perform the corresponding movement.

After a 90 -seconds distractor task (reading a poem), the test phase presented participants with the stimuli of all twelve items for retrieval of the sequential finger 
Inhibition in motor memory

movement, that is, the test format was identical to retrieval practice. Two test sequences were counterbalanced between participants either starting with Rp- items (then $\mathrm{Nrp}-, \mathrm{Rp}+$, and $\mathrm{Nrp}+$ ) or starting with Nrp- items (then Rp-, Nrp+, and Rp+). Thus, the items of one item type were tested in direct succession. Test trials were repeated in ten cycles, that is, after trials for the three items of one type had been presented once, they were repeated nine times before proceeding with test trials for the next item type. This repetition of test trials ensured sufficient reliability of EEG recording (note however that EEG data from the test phase were not analyzed and are not reported in the current manuscript).

After a five-minute break, block 2 started. The items now were another 12 sequential finger movements. The numbers 1 to 6 were stimuli for the left-hand movements, the numbers 7 to 12 were stimuli for the right-hand movements. In the retrieval-practice phase, the response hand changed from block 1 to block 2 . A different poem served as distractor task. In every other respect, the procedure was identical to block 1.

EEG recording. Electrophysiological data were recorded from $65 \mathrm{Ag} / \mathrm{AgCl}$ electrodes, which were positioned according to the international 10-10 electrode system with reference to $\mathrm{FCz}$ (EC80, Montage No. 1, Easycap, Herrsching, Germany). The ground electrode was placed at location Fpz. The electrooculogram (EOG) was recorded from four additional bipolar channels, positioned on the inferior and superior regions of the left eye and the outer canthi of both eyes, in order to monitor the vertical and horizontal EOG. Electrodeskin impedance was kept below $5 \mathrm{k} \Omega$ for all scalp electrodes. Signals were digitalized with a sampling rate of $500 \mathrm{~Hz}$ and amplified between $0.016 \mathrm{~Hz}$ and $250 \mathrm{~Hz}$ (BrainAmp, BrainVision Recorder, v1.20, Brain Products, Gilching, Germany).

EEG preprocessing. EEG recordings were re-referenced offline against average reference and EOG corrected using calibration data and generating individual EOG artifact coefficients, as implemented in BESA Research (v6.1, BESA Software, Gräfelfing, 
Inhibition in motor memory

Germany; see Ille, Berg, \& Scherg, 2002). Remaining artifacts (mainly muscle artifacts and jumps) were marked by careful visual inspection. EEG signals of single channels (65) showing heavy artifacts throughout a single session were interpolated using spline interpolation as implemented in BESA research (mean number of channels $=1.12 ; \mathrm{SD}=$ 2.27). EEG data were segmented into epochs ranging from -1.5 to $2.5 \mathrm{sec}$ around the onset of stimuli, i.e., letter or number cues, in the retrieval-practice phase. Segments containing artifacts were discarded from the analysis; segments of trials with response errors were kept in the analysis (cf. Tempel \& Frings, 2013). Mean number of trials included in EEG data analysis were 26.0 (out of 30 ) of the $1^{\text {st }}$ half $(\mathrm{SD}=3.05$; Min=14) and 25.5 (out of 30 ) of the $2^{\text {nd }}$ half $(\mathrm{SD}=3.39$; Min=13) of trials in the retrieval-practice phase (note that EEG oscillatory [de]synchronization patterns stabilize with about 15 segments per condition; see simulation experiment by Hanslmayr, Spitzer, \& Bäuml, 2009).

Time-frequency decomposition. EEG data were transformed into the time-frequency domain using a complex demodulation algorithm that is implemented in BESA Research (v6.1, see Hoechstetter et al., 2004). The algorithm consists of a multiplication of the timedomain signal with a complex periodic exponential function, having a frequency equal to the frequency under analysis, and subsequent low-pass filtering. The low-pass filter is a finite impulse response filter of Gaussian shape in the time domain, which is related to the envelope of the moving window in wavelet analysis. The EEG data were filtered in a frequency range from 2 to $30 \mathrm{~Hz}$. Time resolution was set to $78.8 \mathrm{msec}$ (full power width at half maximum, FWHM) and frequency resolution was set to $1.42 \mathrm{~Hz}$ (FWHM). Evoked activity was subtracted from all single trials by calculating the average waveform for each channel and subtracting its time-frequency transform from the time-frequency transform of each individual trial; thus, induced power was analyzed (a priori analysis showed that the conclusions were the same without subtraction of the evoked signal, i.e., for total power). 
Inhibition in motor memory

Time-frequency data were exported in bins of $50 \mathrm{msec}$ and $1 \mathrm{~Hz}$. Stimulus-locked changes in EEG power were determined by calculating the temporal-spectral evolution, i.e., percentage power changes for all time-frequency points with power increases or decreases at time point $t$ and frequency f related to mean power at frequency f over a preceding baseline interval (Pfurtscheller \& Aranibar, 1977). The baseline interval that was set from -0.25 to $0 \mathrm{sec}$ timelocked to stimulus onset.

Statistical analysis of time-frequency data. Statistical analysis was restricted to time-frequency data of the retrieval-practice phase. To avoid filter artifacts at the edges of the segmented time-frequency data, statistical analysis of power changes was restricted to the time range from 0 to $1.2 \mathrm{sec}$ following stimulus onset and the frequency range from 4 to 30 Hz. Following Staudigl et al. (2010), power changes were compared between the $1^{\text {st }}$ half (cycles $1-5$ ) and the $2^{\text {nd }}$ half (cycles 6-10) of trials in the retrieval-practice phase. To control for problems of multiple comparisons when testing the significance of power differences over multiple time-frequency points and electrode sites, cluster and random permutation analyses were conducted (Maris \& Oostenveld, 2007), using the software package BESA Statistics (v2.0, BESA Software, Gräfelfing, Germany).

Arguably, cluster-based permutation analysis allows multiple comparison control for establishing a significant difference between conditions, i.e. practice halves, but not for establishing significant differences for individual time bins, frequency bins, or electrodes that are included in a significant cluster (see Maris \& Ostenveld, 2002; Sassenhagen \& Draschkow, 2019). Therefore, different cluster analyses were calculated for the hypothesized theta and beta power effects. For theta, the cluster analyses were restricted from 4 to $10 \mathrm{~Hz}$, whereas for beta the analyses were restricted from 10 to $20 \mathrm{~Hz}$. In addition, cluster analyses for the frequency range from 20 to $30 \mathrm{~Hz}$ were calculated in order to examine possible broadband power effects in the beta/gamma frequency range. Note that due to low spatial 
Inhibition in motor memory

resolution of scalp EEG in general and in order to avoid overestimation of location effects in cluster-based permutation analysis (Maris \& Ostenveld, 2002; Sassenhagen \& Draschkow, 2019), spatial information was not used in the present cluster analyses (see also Maris, 2012, for an overview of key steps in permutation-based cluster analysis).

Two steps were taken in the statistical analysis of the time-frequency data. In the first step, time-frequency data were compared between the $1^{\text {st }}$ half (cycles 1-5) and the $2^{\text {nd }}$ half (cycles 6-10) of trials in the retrieval-practice phase, separately for the frequency ranges from 4 to $10 \mathrm{~Hz}, 10$ to $20 \mathrm{~Hz}$, and 20 to $30 \mathrm{~Hz}$. For each frequency range, a non-spatial cluster analysis was calculated first and a spatial analysis second. In the non-spatial cluster analysis, time-frequency spectrograms of stimulus-locked (induced) power changes were averaged across the 65 electrodes and contrasted between practice halves. Two-tailed $t$ tests were calculated for all time-frequency points. The sum of $t$ values of adjacent time-frequency points that fell below a $\mathrm{p}$ value of .05 in the $t$ test was calculated as a test statistic. Random permutation analysis was calculated based on 10000 randomization runs. In each randomization run, time-frequency data of the two practice halves were interchanged randomly for each participant and $t$ tests were calculated for each time-frequency point. At the end of each run, $t$ values of adjacent time-frequency points that fell below a $p$ value of .05 were summed and the cluster with the highest sum of t values was kept. By these means, a null distribution of cluster sums was created from the 10000 permutation runs, and the critical prand value for an empirically derived time-frequency cluster was estimated. Empirical clusters with a prand value below .05 went into spatial analysis. For each cluster, power changes were averaged across data points of the cluster's maximum time range and maximum frequency range, separately for each electrode. These data were contrasted between practice halves. Two-tailed t tests were calculated for all electrodes. Spatial topographies were identified by considering those electrodes that fell below a $\mathrm{p}$ value of .05 
Inhibition in motor memory

in the $\mathrm{t}$ test. No additional cluster analysis was calculated. Thus, both clustered and scattered effects of practice halves $\left(1^{\text {st }}\right.$ half vs. $2^{\text {nd }}$ half $)$ were considered in the spatial analysis.

In the second step, individual differences in time-frequency data between practice halves were correlated with the behavioral retrieval-induced slowing and acceleration scores, separately for the frequency ranges from 4 to $10 \mathrm{~Hz}, 11$ to $20 \mathrm{~Hz}$, and 21 to $30 \mathrm{~Hz}$. For each frequency range, a non-spatial cluster analysis was calculated first and a spatial analysis second. In the non-spatial cluster analysis, differences in time-frequency data between practice halves ( $2^{\text {nd }}$ half minus $1^{\text {st }}$ half) were averaged across the 65 electrodes and correlated with the behavioral retrieval-induced slowing and acceleration scores by estimating Pearson correlations and calculating two-tailed t tests for all time-frequency points, respectively. Only adjacent time-frequency points that fell below a $\mathrm{p}$ value of .05 in the $\mathrm{t}$ test were considered. For each cluster, the sum of $t$ values was calculated as a test statistic. Calculation of prand was based on 10000 random permutation runs. Empirical clusters with a prand value below .05 went into spatial analysis. For each cluster, power differences were averaged across data points of the cluster's maximum time range and maximum frequency range, separately for each electrode (65). These data were correlated with the retrieval-induced slowing and acceleration scores, respectively. Pearson correlations and two-tailed t tests were calculated for all electrodes. Spatial topographies were identified by considering those electrodes that fell below a p value of .05 in the $t$ test. Due to non-independence between the spatial and the non-spatial analysis, no additional cluster analysis was calculated. Thus, both clustered and scattered correlation effects were considered in the spatial analysis.

\section{Results}

\section{Behavioral Results}

Mean retrieval success in the retrieval-practice phase was $67 \%(S D=30 \%)$. Whereas accuracy did not differ between the two halves of retrieval-practice cycles, $|t|<1$, execution 
Inhibition in motor memory

became faster, $t(47)=7.30, p<0.001$. Mean speed was $2,496 \mathrm{~ms}(S D=806 \mathrm{~ms})$ in the first half and $1,825 \mathrm{~ms}(S D=392 \mathrm{~ms})$ in the second half.

To examine retrieval-induced forgetting and $\mathrm{Rp}+$ enhancement, response times (RTs) and the number of recalled items in the first cycle of test trials were analyzed. Examining the first cycle of test trials, significantly longer RTs for Rp- items than Nrp- items reflected retrieval-induced forgetting, $t(47)=2.53, p=0.015, d_{z}=0.37$. Significantly shorter RTs for $\mathrm{Rp}+$ items than $\mathrm{Nrp}+$ items reflected $\mathrm{Rp}+$ enhancement, $t(47)=2.23, p=0.030, d_{z}=0.32$ (see Table 1). Neither of the two effects was significant with regard to the number of recalled items $|t|<1$. When analyzing performance over all ten test cycles, the behavioral data showed basically the same pattern of results with longer RTs for Rp- items than Nrp- items, $t(47)=$ $1.94, p=0.059, d_{z}=0.28$, as well as shorter RTs for Rp+items than $\mathrm{Nrp}+$ items, $t(47)=$ $3.73, p=0.001, d_{z}=0.54$.

Table 1. Mean $(M)$ proportion of recalled items in the first cycle of the test phase and response times in milliseconds with standard error of the mean $(S E)$ as a function of item type.

\begin{tabular}{|c|c|c|c|c|c|c|c|c|}
\hline & \multicolumn{2}{|c|}{$\mathrm{Rp}-$} & \multicolumn{2}{|c|}{ Nrp- } & \multicolumn{2}{|c|}{$\mathrm{Nrp}+$} & \multicolumn{2}{|c|}{$\mathrm{Rp}+$} \\
\hline & $M$ & $S E$ & $M$ & $S E$ & $M$ & $S E$ & $M$ & $S E$ \\
\hline $\begin{array}{l}\text { Proportion of } \\
\text { recalled items }\end{array}$ & 0.78 & 0.04 & 0.76 & 0.04 & 0.75 & 0.04 & 0.75 & 0.04 \\
\hline $\begin{array}{l}\text { Response } \\
\text { time }\end{array}$ & 2646 & 220 & 2197 & 118 & 2479 & 197 & 2149 & 200 \\
\hline
\end{tabular}


Inhibition in motor memory

\section{EEG Results}

Cluster-based permutation testing of contrasts between practice halves indicated that there were two significant differences between the $1^{\text {st }}$ and the $2^{\text {nd }}$ half of trials in the retrievalpractice phase. One cluster in the observed data showed a decrease of fast (or upper) theta power (approximately 6 to $10 \mathrm{~Hz})$ from the $1^{\text {st }}$ to the $2^{\text {nd }}$ half $\left(p_{\text {rand }}=0.001\right)$; this cluster extended approximately from 100 to $450 \mathrm{~ms}$ after stimulus onset (see Figures 2A \& 2B). Spatial analysis indicated that the effect was most pronounced over central and parietal electrodes (see Figure 2C). In addition, the second cluster analysis showed an increase of beta power (approximately 11 to $18 \mathrm{~Hz})$ from the $1^{\text {st }}$ to the $2^{\text {nd }}$ half $\left(p_{\text {rand }}<0.001\right)$; this cluster extended approximately from 500 to $1200 \mathrm{~ms}$ following stimulus onset (see Figures 2A \& 2B). The effect was most pronounced over frontal and tempo-parietal electrodes (see Figure 2D). Time courses of these effects are shown in Figures 2E and 2F. The third cluster analysis for the frequency range from 20 to $30 \mathrm{~Hz}$ revealed no significant clusters.
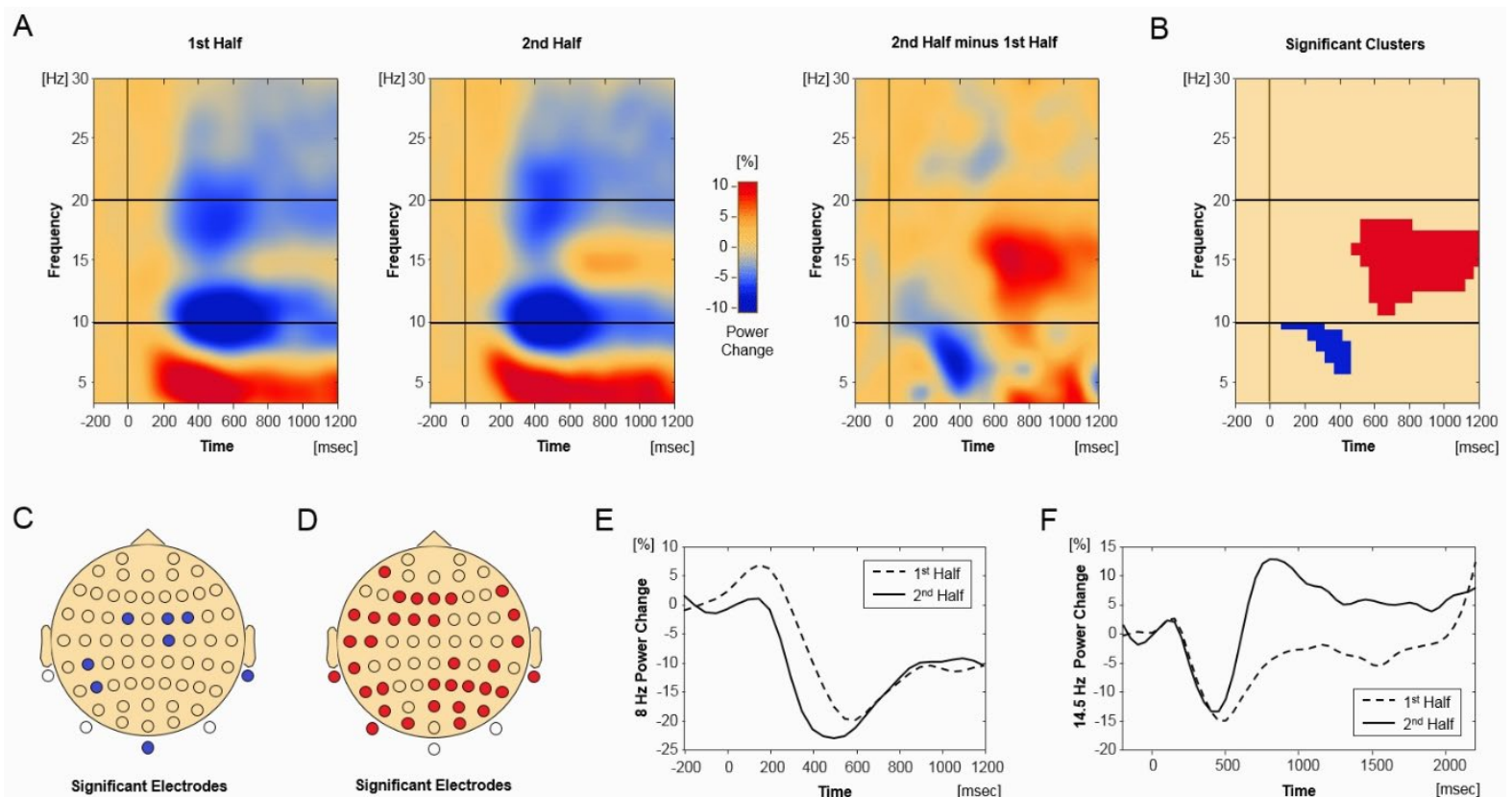

$E$

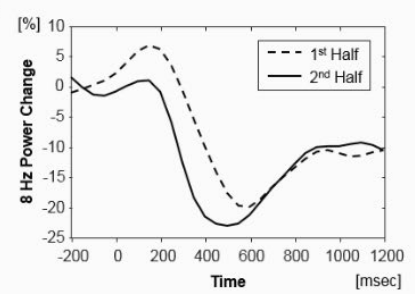

$\mathrm{F}$

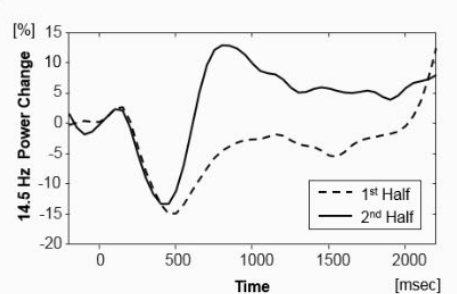


Inhibition in motor memory

Figure 2. EEG results. (A) Increases and decreases of EEG power from the first to the second half of retrievalpractice cycles. (B) Significant clusters. (C) Electrodes with significant decreases of fast (or upper) theta power. (D) Electrodes with significant increases of beta power. (E) Time course of theta power change (at median-effect frequency of $8 \mathrm{~Hz}$ ), separately for the first and second half of retrieval-practice cycles. (F) Time course of beta power change (at median-effect frequency of $14.5 \mathrm{~Hz}$ ), separately for the first and second half of retrievalpractice cycles.

Cluster-based permutation analyses of correlations between differences in timefrequency data ( $2^{\text {nd }}$ half minus $1^{\text {st }}$ half of trials in the retrieval-practice phase) and the behavioral retrieval-induced slowing and acceleration scores were calculated separately for the frequency ranges from 4 to $10 \mathrm{~Hz}, 10$ to $20 \mathrm{~Hz}$, and 20 to $30 \mathrm{~Hz}$. The score for retrievalinduced slowing was calculated as the difference between Nrp- and Rp- Items, the score for retrieval-induced acceleration was calculated as the difference between Rp+ and Nrp+ items. The analyses showed a single significant cluster regarding the correlation with slowing ( $\mathrm{p}_{\text {rand }}$ $=0.033$; all other clusters, $p_{\text {rand }}>0.220$ ) but no significant cluster regarding the correlation with acceleration (all $p_{\text {rand }}>0.320$ ). The cluster in the observed data showed a positive correlation with the slowing score in the beta frequency range (approximately 11 to $16 \mathrm{~Hz}$ ); this cluster extended approximately from 550 to $1100 \mathrm{~ms}$ following stimulus onset (see Figures 3A \& 3B). Spatial analysis indicated that the effect was most pronounced over left frontal and left tempo-parietal electrodes (see Figure 3C). 
A

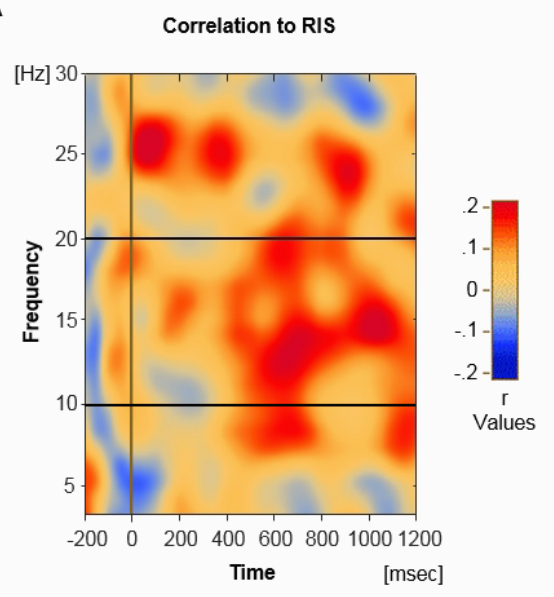

B

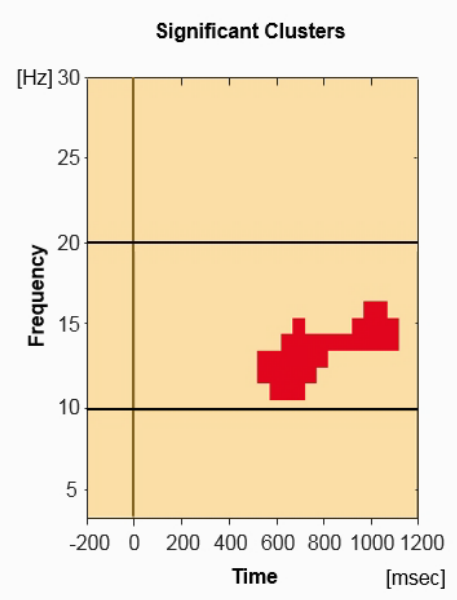

C

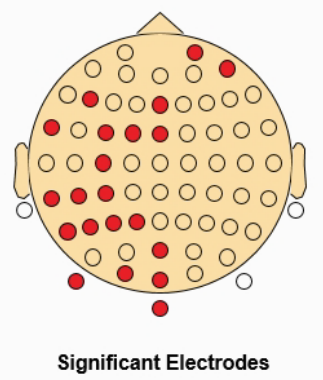

Figure 3. Correlations between EEG power changes during retrieval practice ( $2^{\text {nd }}$ half minus $1^{\text {st }}$ half $)$ and retrieval-induced-slowing scores. (A) Correlation (r) values as function of time and frequency. (B) Significant cluster. (C) Electrodes with significant correlations.

\section{Discussion}

An increase in beta power during selective retrieval practice predicted subsequent retrieval-induced slowing of motor sequences. This observation matches the inhibition theory of retrieval-induced forgetting that posits that forgetting occurs as a consequence of an inhibitory mechanism that directly affects $\mathrm{Rp}$ - items during selective retrieval practice of $\mathrm{Rp}+$ items. In correspondence with this assumption, we found that a slowed-down execution of Rp- items in the final test phase was uniquely predicted by the beta-power increase during retrieval practice, which was independent from strengthening of the retrieval-practiced $\mathrm{Rp}+$ items. Although Rp+ items were strengthened by retrieval practice, which was reflected by significant acceleration of movement execution in the final test phase, this acceleration was unrelated to the beta-power increase. Thus, brain-behavior correlations suggest that this particular oscillatory pattern relates to a process that is specific for the slowing effect. A noninhibitory theory that would assume blocking by strengthened $\mathrm{Rp}+$ items to account for retrieval-induced forgetting (Raaijmakers \& Jakab, 2013), therefore, is not able to explain the 
Inhibition in motor memory

present finding of significant brain-behavior correlation of beta-power increase during retrieval practice with retrieval-induced slowing for RP- items but not with retrieval-induced fastening for RP+ items.

As a further alternative to the inhibition theory aside from blocking, a mental context change occurring at retrieval practice has been suggested (Jonker et al., 2013). Similarly to inhibition theory, this model posits that a process during retrieval practice that is independent from strengthening of $\mathrm{Rp}+$ items accounts for retrieval-induced forgetting. Might, perhaps, beta-power increase reflect a mental-context change instead of an inhibitory mechanisms triggered by retrieval practice? Several aspects speak against this possibility. First, the mental context change is assumed to occur when transitioning from the study phase to retrieval practice. It is triggered by the novel task demands. Thus, a physiological marker of mentalcontext change should be strongest around that transition and weaken subsequently. However, beta power did increase over the course of retrieval practice in the present investigation, not decline, as might be predicted by the context-change account. In accordance with the inhibition theory, this could suggest that inhibition intensified over repeated retrieval-practice cycles. In contrast, theta power did decline over the course of retrieval practice and thus may be linked to context change in the predicted manner. However, theta power was not related to behavior during final recall testing. Second, we are not aware of previous studies that have linked beta power increase to context change in general and mental context changes in particular, whereas a great number of studies suggest beta power increase to reflect inhibition in different cognitive domains (e.g. Alegre et al., 2004; Castiglione, Wagner, Anderson, \& Aron, 2019; Pastötter et al., 2008; Wheaton et al., 2009). Third, recent behavioral findings have raised serious doubts on the context-change theory of retrieval-induced forgetting, especially with regard to the replicability of evidence 
Inhibition in motor memory

that had been reported as evidence of this theory (Buchli, Storm, \& Bjork, 2016; Rupprecht \& Bäuml, 2017).

Taken together, the inhibition theory is able to explain the present findings better than current alternatives. We had chosen motor sequences as the item material here because they promised to show effects on beta power increase that had been found previously to be a physiological marker of response inhibition (e.g. Pastötter et al., 2008; Swann et al., 2009; Wheaton et al., 2009). Here, this measure was found to accompany an effect that regularly has been assumed to arise from inhibition in memory. Although, the number of correct responses per item type was not reliably affected by retrieval practice, retrieval-induced forgetting occurred here in the form of retrieval-induced slowing. In fact, the speed of movement execution has been found to be affected by selective retrieval practice more consistently than recall accuracy in previous studies (Tempel \& Frings, 2013, 2016). The present pattern of results further substantiates that theory: Retrieval practice triggers inhibition also in motor memory. Whereas beta power increase is a well-established marker of response inhibition, it has also been linked to inhibition in declarative memory in a few studies. For example, a recent study by Castiglione et al. (2019) that found an increase in EEG beta power during instructed thought suppression in the think/no-think paradigm (Anderson \& Green, 2001). Preventing a thought from coming to mind apparently involved the same kind of inhibitory power as stopping an action did. Yet, it remained unclear whether this inhibitory mechanism actually affected item memory because beta power increase was not correlated with participants' suppression-induced forgetting score. By contrast, in the present study, we found that beta power increase during selective memory retrieval predicted subsequent retrieval-induced forgetting. Possibly, in thought suppression, increased beta power reflects inhibitory power that is directed at preventing a thought from entering consciousness but is not linked to the individual accessibility of specific items. Definitely, 
Inhibition in motor memory

more future research is needed to compare the role of beta power increase in retrievalinduced forgetting and instructed thought suppression more directly.

Consistent with earlier work on declarative memory (Hanslmayr et al., 2010; Pastötter \& Bäuml, 2014; Staudigl et al., 2010) and non-declarative, visual memory (Waldhauser et al., 2012), the present results revealed an interference-related effect in theta power for motor memory. In line with the study by Pastötter and Bäuml (2014), the effect was observed in the fast (or upper) theta frequency range. Regarding retrieval-induced inhibition of visual memories, Waldhauser et al. (2012) examined lateralized EEG power effects during selective retrieval of relevant target information (i.e., color coding of the relevant patches) that was studied together with irrelevant distractor information (i.e., color coding of the irrelevant patches) when using lateralized presentation of visual stimuli (i.e., simultaneous presentation of lateralized color patches that were associated with meaningless objects). During selective retrieval, reliable EEG alpha/beta power increase was observed contralateral to distractor presentation side. This finding extends previous findings from visual attention studies (e.g., Worden et al., 2000; Kelly et al., 2006; Sauseng et al., 2009), suggesting that the inhibitory mechanisms regulating visual information selection generalize from visual attention to visual memory. In contrast to the study by Waldhauser et al. (2012), the present study examined whether the link between EEG beta power increase and motor response inhibition generalizes to inhibition in motor memory.

The use of motor sequences as item material (in contrast to the typical word material) does not just allow for a demonstration of how basic the phenomenon of retrieval-induced forgetting is, affecting behavior and cognition across domains. It also opens new research approaches that investigate the processes contributing to the observed phenomena. Combining EEG methods with motor material, our results suggest that beta power increase 
Inhibition in motor memory

might be a general marker of inhibition across cognitive domains, operating in motor control, perception, and memory.

Acknowledgements

This research was supported by grant TE 891/3-3 of the German Research Council (DFG). 
Inhibition in motor memory

References

Alegre, M., Gurtubay, I. G., Labarga, A., Iriarte, J., Valencia, M., \& Artieda, J. (2004). Frontal and central oscillatory changes related to different aspects of the motor process: A study in go/no-go paradigms. Experimental Brain Research, 159, 14-22. DOI: $10.1007 / \mathrm{s} 00221-004-1928-8$

Anderson, M. C. (2003). Rethinking interference theory: Executive control and the mechanisms of forgetting. Journal of Memory and Language, 49, 415-445. DOI: 10.1016/j.jml.2003.08.006

Anderson, M. C., Bjork, R. A., \& Bjork, E. L. (1994). Remembering can cause forgetting: Retrieval dynamics in long-term memory. Journal of Experimental Psychology: Learning, Memory, and Cognition, 20, 1063-1087. DOI: 10.1037/02787393.20.5.1063

Anderson, M.C., \& Green, C. (2001). Suppressing unwanted memories by executive control. Nature, 410, 366-369. DOI: 10.1038/35066572

Bäuml, K.-H., Pastötter, B., \& Hanslmayr, S. (2010). Binding and inhibition in episodic memory - Cognitive, emotional, and neural processes. Neuroscience and Biobehavioral Reviews, 34, 1047-1054. DOI: 10.1016/j.neubiorev.2009.04.005

Buchli, D., Storm, B. C., \& Bjork, R. A. (2016). Explaining retrieval-induced forgetting: A change in mental context between the study and restudy practice phases is not sufficient to cause forgetting. The Quarterly Journal of Experimental Psychology, 69, 1197-1209. DOI: 10.1080/17470218.2015.1076866

Castiglione, A., Wagner, J., Anderson, M., Aron, A. R. (2019). Preventing a thought from coming to mind elicits increased right frontal beta just as stopping action does. Cerebral Cortex, 29, 2160-2172. DOI: 10.1093/cercor/bhz017

Friehs, M., \& Frings, C. (2018). Pimping Inhibition: Anodal tDCS enhances Stop-Signal 
Inhibition in motor memory

Reaction Time. Journal of Experimental Psychology: Human Perception and Performance, 44(12), 1933-1945. DOI: 10.1037/xhp0000579

Hanslmayr, S., Spitzer, B., \& Bäuml, K. H. (2009). Brain oscillations dissociate between semantic and nonsemantic encoding of episodic memories. Cerebral Cortex, 19, 1631-1640. DOI: 10.1093/cercor/bhn197

Hanslmayr, S., Staudigl, T., Aslan, A., \& Bäuml, K.-H. (2010). Theta oscillations predict the detrimental effects of memory retrieval. Cognitive, Affective, \& Behavioral Neuroscience, 10, 329-338. DOI: 10.3758/CABN.10.3.329

Hoechstetter, K., Bornfleth, H., Weckesser, D., Ille, N., Berg, P., \& Scherg, M. (2004). BESA source coherence: A new method to study cortical oscillatory coupling. Brain Topography, 16, 233-238. DOI: 10.1023/B:BRAT.0000032857.55223.5d

Ille, N., Berg, P., \& Scherg, M. (2002). Artifact correction of the ongoing EEG using spatial filters based on artifact and brain signal topographies. Journal of Clinical Neurophysiology, 19, 113-124. DOI: 10.1097/00004691-200203000-00002

Jonker, T. R., Seli, P., \& MacLeod, C. M. (2013). Putting retrieval-induced forgetting in context: An inhibition-free, context-based account. Psychological Bulletin, 120, 852872. DOI: $10.1037 / \mathrm{a} 0034246$

Kelly, S.P., Lalor, E.C., Reilly, R.B., \& Foxe, J.J. (2006) Increases in alpha oscillatory power reflect an active retinotopic mechanism for distracter suppression during sustained visuospatial attention. Journal of Neurophysiology, 95, 3844-3851. DOI: 10.1152/jn.01234.2005

Kuhl, B. A., Dudukovic, N. M., Kahn, I., \& Wagner, A. D. (2007). Decreased demands on cognitive control reveal the neural processing benefits of forgetting. Nature Neuroscience, 10, 908-914. DOI: 10.1038/nn1918

Maris, E. (2012). Statistical testing in electrophysiological studies. Psychophysiology, 49, 
Inhibition in motor memory

549-565. DOI: 10.1111/j.1469-8986.2011.01320.x

Maris, E., \& Oostenveld, R. (2007). Nonparametric statistical testing of EEG- and MEG-data. Journal of Neuroscience Methods, 164, 177- 190. DOI:

10.1016/j.jneumeth.2007.03.024

Murayama, K., Miyatsu, T., Buchli, D., \& Storm, B. C. (2014). Forgetting as a consequence of retrieval: A meta-analytic review of retrieval-induced forgetting. Psychological Bulletin, 140, 1383-1409. DOI: 10.1037/a0037505

Nigbur, R., Ivanova, G., \& Stürmer, B. (2011). Theta power as a marker for cognitive interference. Clinical Neurophysiology, 122, 2185-2194. DOI:

10.1016/j.clinph.2011.03.030

Pastötter, B., \& Bäuml, K.-H. T. (2014). Distinct slow and fast cortical theta dynamics in episodic memory retrieval. NeuroImage, 94, 155-161. DOI:

10.1016/j.neuroimage.2014.03.002

Pastötter, B., Hanslmayr, S., \& Bäuml, K.-H. (2008). Inhibition of return arises from inhibition of response processes: An analysis of oscillatory beta activity. Journal of Cognitive Neuroscience, 20, 65-75. DOI: 10.1162/jocn.2008.20010

Pfurtscheller, G., \& Aranibar, A. (1977). Event-related cortical desynchronization detected by power measurements of scalp EEG. Electroencephalography and Clinical Neurophysiology, 42, 817-826. DOI: 10.1016/0013-4694(77)90235-8

Raaijmakers, J. G. W. (2018). Retrieval-induced forgetting without competition: Testing the retrieval specificity assumption of the inhibition theory. In John T. Wixted (Ed.), Stevens' Handbook of Experimental Psychology and Cognitive Neuroscience (p.251284). New York: Wiley. DOI: 10.1002/9781119170174.epcn108 
Inhibition in motor memory

Raaijmakers, J. G. W., \& Jakab, E. (2013). Rethinking inhibition theory: On the problematic status of the inhibition theory for forgetting. Journal of Memory and Language, 68, 98-122. DOI: $10.1016 / j . j m l .2012 .10 .002$

Reppa, I., Worth, E. R., Greville, W. J., \& Saunders, J. (2103). The representation of response effector and response location in episodic memory for newly acquired actions: Evidence from retrieval-induced forgetting. Acta Psychologica, 143, 210217. DOI: $10.1016 /$ j.actpsy.2013.03.007

Roediger, H. L. III, \& Schmidt, S. R. (1980). Output interference in the recall of categorized and paired associate lists. Journal of Experimental Psychology: Human Learning and Memory, 6, 91-105. DOI: 10.1037/0278-7393.6.1.91

Rupprecht, J., \& Bäuml, K.-H. (2017). Retrieval-induced versus context-induced forgetting: Can restudy preceded by context change simulate retrieval-induced forgetting? Journal of Memory and Language, 93, 259-275. DOI: 10.1016/j.jm1.2016.10.006

Sassenhagen, J., \& Draschkow, D. (2019). Cluster-based permutation tests of MEG/EEG data do not establish significance of effect latency or location. Psychophysiology, 56, e13335. DOI: $10.1111 /$ psyp.13335

Sauseng, P., Klimesch, W., Heise, K.F., Gruber, W.R., Holz, E., Karim, A.A., Glennon M., Gerloff, C., Birbaumer, N., \& Hummel, F.C. (2009) Brain oscillatory substrates of visual short-term memory capacity. Current Biology, 19, 1846 -1852. DOI: 10.1016/j.cub.2009.08.062

Smith, A. D. (1971). Output interference and organized recall from long-term memory. Journal of Verbal Learning and Verbal Behavior, 10, 400-408. DOI: 10.1016/S00225371(71)80039-7 
Inhibition in motor memory

Staudigl, T., Hanslmayr, S., \& Bäuml, K.-H. T. (2010). Theta oscillations reflect the dynamics of interference in episodic memory retrieval. Journal of Neuroscience, 30, 11356-11362. DOI: 10.1523/JNEUROSCI.0637-10.2010

Swann, N., Tandon, N., Canolty, R., Ellmore, T. M., McEvoy, L. K., Dreyer, S., \& Aron, A. R. (2009). Intracranial EEG reveals a time- and frequency-specific role for the right inferior frontal gyrus and primary motor cortex in stopping initiated responses. The Journal of Neuro-science, 29, 12675-12685. DOI: 10.1523/JNEUROSCI.335909.2009

Tempel, T., Aslan, A., \& Frings, C. (2016). Competition dependence of retrieval-induced forgetting in motor memory. Memory \& Cognition, 44, 671-680. DOI: $10.3758 / \mathrm{s} 13421-015-0578-3$

Tempel, T., \& Frings, C. (2013). Resolving interference between body movements: Retrieval-induced forgetting of motor sequences. Journal of Experimental Psychology: Learning, Memory, and Cognition, 39, 1152-1161. DOI: $10.1037 / \mathrm{a} 0030336$

Tempel, T., \& Frings, C. (2014a). Interference within hands: Retrieval-induced forgetting of left and right hand movements. Acta Psychologica, 148, 1-5. DOI: 10.1016/j.actpsy.2014.01.003

Tempel, T., \& Frings, C. (2014b). Forgetting motor programmes: Retrieval dynamics in procedural memory. Memory, 22, 1116-1125. DOI: 10.1080/09658211.2013.871293

Tempel, T., \& Frings, C. (2015). Categorization by movement direction: Retrieval-induced forgetting of motor sequences grouped by motion features. Quarterly Journal of Experimental Psychology, 68, 473-486. DOI: 10.1080/17470218.2014.945098

Tempel, T., \& Frings, C. (2016). How motor practice shapes memory: Retrieval but not extra study can cause forgetting. Memory, 24, 903-915. DOI: 
Inhibition in motor memory

$10.1080 / 09658211.2015 .1059858$

Tempel, T., \& Frings, C. (2017). Retrieval-induced forgetting is retrieval-modality specific:

Evidence from motor memory. Cognition, 162, 143-152. DOI:

10.1016/j.cognition.2017.02.005

Tempel, T., Loran, I., \& Frings, C. (2015). Dancing your moves away: How memory retrieval shapes complex motor action. Journal of Experimental Psychology: Applied, 21, 300-312. DOI: 10.1037/xap0000052

Verbruggen, F., \& Logan, G. D. (2008). Response inhibition in the stop-signal paradigm. Trends in Cognitive Sciences, 12, 418-424. DOI: 10.1016/j.tics.2008.07.005

Waldhauser, G. T., Johansson, M., \& Hanslmayr, S. (2012). Alpha/beta oscillations indicate inhibition of interfering visual memories. Journal of Neuroscience, 32, 1953-1961. DOI: $10.1523 / J N E U R O S C I .4201-11.2012$

Wessel, J. R. (2018). Prepotent motor activity and inhibitory control demands in different variants of the go/no-go paradigm. Psychophysiology, 55, 1-14. DOI: $10.1111 /$ psyp. 12871

Wheaton, L., Fridman, E., Bohlhalter, S., Vorbach, S., \& Hallett, M. (2009). Left parietal activa-tion related to planning, executing and suppressing praxis hand movements. Clinical Neurophysiology, 120, 980-996. DOI: 10.1016/j.clinph.2009.02.161

Wimber, M., Rutschmann, R. M., Greenlee, M. W., \& Bäuml, K. H. (2009). Retrieval from episodic memory: Neural mechanisms of interference resolution. Journal of Cognitive Neuroscience, 21, 538-549. DOI: 10.1162/jocn.2009.21043

Wohldmann, E. L., Healy, A. F., \& Bourne, L. E., Jr. (2008). A mental practice superiority effect: Less retroactive interference and more transfer than physical practice. Journal of Experimental Psychology: Learning, Memory, and Cognition, 34, 823-833. DOI:10.1037/0278-7393.34.4.823 
Inhibition in motor memory

Worden, M.S., Foxe, J.J., Wang, N., \& Simpson, G.V. (2000) Anticipatory biasing of visuospatial attention indexed by retinotopically specific alpha-band electroencephalography increases over occipital cortex. The Journal of Neuroscience, 20, RC63. DOI: 10.1523/JNEUROSCI.20-06-j0002.2000

Wühr, P., \& Frings, C. (2008). A case for inhibition: Visual attention suppresses the processing of irrelevant objects. Journal of Experimental Psychology: General, 137, 116-130. DOI: 10.1037/0096-3445.137.1.116 
Appendix

Items.

\begin{tabular}{lll}
\hline Category & Sequence & stimulus \\
\hline Left & left, right, middle & $\mathrm{a}$ \\
Left & right, left, right & $\mathrm{b}$ \\
Left & middle, left, right & $\mathrm{c}$ \\
Left & left, middle, left & $\mathrm{d}$ \\
Left & right, middle, left & $\mathrm{e}$ \\
Left & middle, right, middle & $\mathrm{f}$ \\
Right & right, middle, right & $\mathrm{u}$ \\
Right & left, right, left & $\mathrm{v}$ \\
Right & middle, right, left & $\mathrm{W}$ \\
Right & right, left, middle & $\mathrm{x}$ \\
Right & left, middle, right & $\mathrm{y}$ \\
Right & middle, left, middle & $\mathrm{z}$ \\
Left & right, left, middle & 1 \\
Left & left, middle, right & 2 \\
Left & middle, left, middle & 3 \\
Left & right, middle, right & 4 \\
Left & left, right, left & 5 \\
Left & middle, right, left & 6 \\
Right & left, middle, left & 7 \\
Right & right, middle, left & 8 \\
Right & middle, right, middle & 9 \\
Right & left, right, middle & 10 \\
Right & right, left, right \\
Right & middle, left, right & 12 \\
\hline & & \\
\hline
\end{tabular}

